\title{
Proteomic and Immunological Identification of Diagnostic Antigens from Spirometra erinaceieuropaei Plerocercoid
}

\author{
Yan Lu' 10 , Jia-Hui Sun', Li-Li Lu², Jia-Xu Chen', Peng Song', Lin Ai', Yu-Chun Cai', Lan-Hua Li', \\ Shao-Hong Chen ${ }^{1, *}$ (10 \\ ${ }^{1}$ National Institute of Parasitic Diseases, Chinese Center for Disease Control and Prevention (Chinese Center for Tropical Diseases Research); WHO \\ Collaborating Center for Tropical Diseases; NHC Key Laboratory of Parasite and Vector Biology (National Institute of Parasitic Diseases, Chinese \\ Center for Disease Control and Prevention); National Center for International Research on Tropical Diseases; Shanghai, P. R. China; ${ }^{2} T$ he Third \\ Hospital of Shijiazhuang City, Shijiazhuang, P. R. China; ${ }^{3}$ School of Public Health, Weifang Medical University, Weifang, P. R. China
}

\begin{abstract}
Human sparganosis is a food-borne parasitic disease caused by the plerocercoids of Spirometra species. Clinical diagnosis of sparganosis is crucial for effective treatment, thus it is important to identify sensitive and specific antigens of plerocercoids. The aim of the current study was to identify and characterize the immunogenic proteins of Spirometra erinaceieuropaei plerocercoids that were recognized by patient sera. Crude soluble extract of the plerocercoids were separated using 2-dimensional gel electrophoresis coupled with immunoblot and mass spectrometry analysis. Based on immunoblotting patterns and mass spectrometry results, 8 antigenic proteins were identified from the plerocercoid. Among the proteins, cysteine protease protein might be developed as an antigen for diagnosis of sparganosis.
\end{abstract}

Key words: Spirometra erinaceieuropaei, plerocercoid, immunoproteomic, diagnosis, antigen

\section{INTRODUCTION}

Human sparganosis is a food-borne zoonosis mainly caused by the plerocercoids (spargana) of various diphyllobothroid tapeworms belonging to the genus Spirometra [1,2]. Human beings mostly serve as the intermediate host, paratenic host, and even the definitive host of Spirometra species in extremely rare cases [3]. Drinking raw water contaminated with cyclops harboring procercoids, ingesting undercooked meat of frogs or snakes infected with spargana, or placing poultices of frog or snake flesh/skin on the eyes or open wounds and other lesions are important sources of human infections $[1,2,4]$.

Sparganosis is distributed worldwide, but the majority of cases occurred in East and Southeast Asian countries, including South China, Korea, Thailand, and Japan [1-3,5-9]. More than 2,000 human sparganosis cases have been reported globally, with more than $80 \%$ of the reported cases from China $[1,2]$, followed by Korea [3-5], Thailand [6,7] and Japan [8,9]. Since the first case of human sparganosis was reported in 1882

\footnotetext{
- Received 22 July 2021, revised 27 November 2021, accepted 27 November 2021. *Corresponding author (chensh@nipd.chinacdc.cn) (C) 2021, Korean Society for Parasitology and Tropical Medicine This is an Open Access article distributed under the terms of the Creative Commons Attribution Non-Commercial License (https://creativecommons.org/licenses/by-nc/4.0) which permits unrestricted non-commercial use, distribution, and reproduction in any medium, provided the original work is properly cited.
}

in Xiamen of Fujian Province, China [10], a total of more than 1,300 cases of human sparganosis have been reported in 27 provinces in mainland China $[1,11]$. But the actual number of human infections may be far higher than those estimated because many cases may not be recognized or reported. Thus, sparganosis has become an emerging food borne parasitic diseases and poses a serious threat to human health [12].

Sparganosis can cause serious clinical problems in human according to the location of the parasites. Plerocercoids can invade subcutaneous tissue, muscle, eye, face, neck, breast, pleural cavity, lung, urogenital viscera, abdominal viscera, brain or central nervous system, resulting in subcutaneous sparganosis, ocular sparganosis, cerebral sparganosis, visceral sparganosis, or proliferative sparganosis $[1-3,13]$. The clinical symptoms of human sparganosis varying from non-specific discomfort, subcutaneous nodules, irritation, continued foreign body sensation, severe inflammation with blindness, headache, seizure, weakness, hemiparesis, abdominal pain, chest pain, pleural effusion, and eosinophilia [14-19].

Although the detection of a plerocercoid is the gold standard method for diagnosing sparganosis, the diagnosis of sparganosis is rather difficult and is often misdiagnosed because the worm has no predilection for particular sites in the human body and symptomatology is not pathognomonic [1, 19]. A diagnosis of subcutaneous sparganosis can be made by 
detection of the worm in a biopsy specimen, but the confirmative diagnosis is very difficult for visceral and cerebral sparganosis since the worm can be found only by surgical removal and sometimes surgical treatment is impossible $[19,20]$. Imaging techniques including ultrasonography, computed tomography (CT) and magnetic resonance imaging (MRI) are usually used for the preoperative diagnosis of sparganosis affecting the eye, brain, spinal cord, abdomen, and other visceral organs $[13,21-23]$. However, the imaging findings are complicated, and misdiagnosis is unavoidable using these techniques as sparganosis is confused with tumors, inflammatory granulo$\mathrm{ma}$, varicose vein or conus medullaris $[24,25]$. Therefore, development of sensitive and specific immunological techniques would accelerate the process of detection and treatment of the sparganosis and subsequently avoid serious complications. Among the serological tests, ELISA with crude antigens or excretory-secretory (ES) antigens of plerocercoids has high sensitivity for the detection of specific antibodies [26-28]. The main disadvantage is cross-reactions with the sera from patients with clonorchiasis, cysticercosis, or paragonimiasis [26,29]. Furthermore, the preparation of crude antigens or ES antigens requires plerocercoids collected from naturally infected hosts or experimentally infected laboratory animals, which is practically inconvenient in terms of cost, labor, and time. Hence, studies on the sensitive and specific recombinant antigens will further improve the diagnosis and treatment of sparganosis.

The combination of 2-dimensional electrophoresis (2-DE) with western blotting help support discovery of numerous novel antigens and the screening of novel serological diagnostic markers and vaccine candidates of parasites. When used together with mass spectrometry, the techniques enable the identification of the proteins that induce immune response and which could be used for immunodiagnosis. This immunoproteomics tool has previously been used to determine both the characteristics of the serological response directed against parasites and immunogenic proteins, such as Schistosoma japonicum [30], Ascaris lumbricoides [31], Trichinella species [32], Toxoplasma gondii [33], and Babesia mocroti [34]. Several studies have analyzed the $S$. erinaceieuropaei antigenic proteins recognized by sera of infected mice using immunoproteomics and mass spectrometry $[35,36]$. However, the immunogenic proteins of plerocercoids have not been well studied previously.

The aim of present study was to identify and characterize the immunogenic proteins of $S$. erinaceieuropaei that were recognized by a pooled serum samples of patients with sparganosis.

\section{MATERIALS AND METHODS}

\section{Ethics statement}

The study and collection of serum specimens were granted by the Ethics Committee of the National Institute of Parasitic Diseases, Chinese Center for Disease Control and Prevention. (Ref No. 20170510). A written informed consent was obtained from each patient or their proxy.

\section{Sample collection}

The plerocercoids of $S$. erinaceieuropaei in this study were collected from subcutaneous tissues and muscles of infected snakes (Zaocys dhumnades), which were submitted for detection by Shanghai Zoo located in the P.R. China. The plerocercoids were washed 5 times with sterilized saline to remove the host debris and cells, and then identified by microscopic analysis and sequencing analysis, as reported previously [37].

\section{Serum samples}

The serum samples of patients with sparganosis, echinococcosis, cysticercosis, trichinellosis, schistosomiasis, clonorchiasis and paragonimiasis were obtained (each $n=10$ ) from the sera bank of National Institute of Parasitic Diseases, Chinese Center for Disease Control and Prevention. The diagnosis of sparganosis patients was confirmed by pathological examination of biopsy specimen or identification of worm body (Supplementary Table S1). Other parasitic infections were diagnosed on the basis of clinical manifestations and positive parasitological examination. Sera from people free of the above-mentioned parasitic infections were included as normal controls.

\section{Preparation of soluble extract from S. erinaceieuropaei plerocercoids}

The plerocercoids were suspended in a lysis buffer (4\% SDS, $100 \mathrm{mM}$ Tris-HCl, 1 mM DTT, pH 7.6), homogenized with a tissue grinder, repeated freezing and thawing 3 times, and then sonicated with 3 cycles at $80 \mathrm{~Hz}$ for $60 \mathrm{sec}$ each on ice. The homogenate was centrifuged at $12,000 \mathrm{~g}$ for $1 \mathrm{hr}$ at $4^{\circ} \mathrm{C}$ and the supernatant was taken as soluble extract. Protein concentration was determined using Bradford assay. The extracts were stored at $-80^{\circ} \mathrm{C}$ until use.

\section{2-DE}

The soluble extracts were separated by 2-DE as described previously [30]. Briefly, 2-dimensional separation gels were performed in quadruplicate; $80 \mu \mathrm{g}$ and $800 \mu \mathrm{g}$ soluble extracts 
were loaded onto analytical gel and preparative gels, respectively. Isoelectric Focusing (IEF) was performed in $18 \mathrm{~cm} \mathrm{Im-}$ mobiline DryStrip with pH 4-7 (GE Healthcare, Pittsburgh, Pennsylvania, USA) and using Ettan IPGphor 3 apparatus (GE Healthcare). The soluble extracts were mixed with rehydration buffer. The second dimension electrophoresis was performed on $12.5 \%$ SDS-PAGE with the ETTAN DALTsix (GE Healthcare). The analytical gel was dyed with silver, one preparative gel was dyed with Coomassie brilliant blue R-250 solution whereas the other gels were processed for further western blot analysis.

\section{Western blot analysis}

Proteins from 2-DE gels were electrotransferred synchronously to 2 nitrocellulose membranes (GE Healthcare). The 2 membranes were blocked in Tris-buffered saline with $0.1 \%$ Tween-20 (TBST, $0.01 \mathrm{~mol} / \mathrm{L}$, pH7.4) containing 5\% skimmed milk at $4^{\circ} \mathrm{C}$ overnights. Then, one membrane was incubated in the pooled sera of sparganosis patients (1:500 diluted), the other membrane in the pooled sera of healthy controls. The secondary antibody HRP-conjugated goat anti-human IgG (Sigma-Aldrich, St. Louis, Missouri, USA) were diluted 1: 8,000 . The immunoreactive spots were visualized on films using the enhanced chemiluminesce (ECL) Western Blot kit (Thermo Fisher Scientific, Frederick, Maryland, USA). The membranes were scanned with Tanon 5200 Multi chemiluminescent image system (Tanon, Shanghai, China) and analyzed with ImageMaster 2D platinum 5.0 (GE Healthcare). Protein spots matching to the immunoblot were manually excised from the preparative gel, and subjected to MS analysis. The electrophoresis and western blot were triplicated to verify immune recognition.

\section{LC-MS/MS and data analysis}

The excised gel pieces carrying the immunoreactive spots were digested with trypsin and the peptides processed as previously described [31]. LC-MS/MS analysis was performed on a Dionex U3000 RSLC nano-LC-system coupled to a Q Exactive mass spectrometer (Thermo Fisher Scientific).

The LC-MS/MS data were analyzed using MASCOT 2.3 (Matrix Science, Boston, Massachusetts, USA) and submitted to MASCOT MS/MS Ions Search server (http://www.matrixscience. $\mathrm{com} /$ ) for identification against NCBI non-redundant protein database (Spirometra erinaceieuropaei, 2021-02-04). Proteins with scores above the significant threshold $(P<0.05)$ were ac- cepted as identified proteins.

\section{Bioinformatics analysis on identified proteins}

The proteins identified in the MASCOT search were assigned to the QuickGO (http://www.ebi.ac.uk/QuickGO/). Then, the proteins classified in gene ontology (GO) in accordance with their biological process, cellular component, and molecular function information.

\section{Preliminary validation of antigenic protein}

Referring to the results of the identification of immunogenic protein, cDNA sequence of cysteine protease (GenBank accession No. BAB62718.1) was synthesized (GeneCreate Biological Engineering Co., Ltd., Wuhan, China) and cloned into pET SUMO vector. The recombinant cysteine protease $(\mathrm{rCP})$ was expressed as a fusion to SUMO-tag (SUMO-rCP) in E. coli BL21 (DE3) and purified using His-Trap FF (GE Healthcare). SUMO-rCP was cleaved by SUMO protease (Solarbio, Beijing, China) according to the manufacturer's instructions. The rCP was purified from cleaved product using nickel-chelating affinity chromatography. The purified $\mathrm{rCP}$ protein was separated by SDS-PAGE, transferred onto nitrocellulose membranes (GE Healthcare), followed by blocking with 3\% BSA in PBS. The membranes were cut into strips, incubated with the pooled patient sera (1:100 dilution) and secondary HRP-conjugated goat anti-human IgG (1:4,000 dilution, Sigma-Aldrich). The immunoblots were stained with DAB (Sigma-Aldrich). The strips were recorded using the GelDoc XR+ Imaging System (Bio-Rad, Hercules, California, USA).

\section{RESULTS}

\section{2-DE}

A total of 245 spots were identified between molecular weight 5-95 kDa and pI 4-7. Most of them were in $25-65 \mathrm{kDa}$ and pI 4.4-6 (Fig. 1A).

\section{Western blot}

Compared with control sera, 8 spots showed up in the pooled sera of sparganosis patients (Fig. 1). These reactive spots matched to the protein spots on Coomassie blue stained gel were excised for further LC-MS/MS analyses.

\section{MS/MS results}

Eight proteins potential antigenic character were assigned to 
A

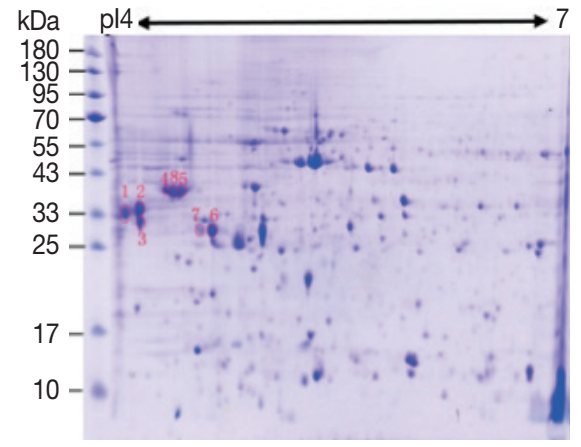

B

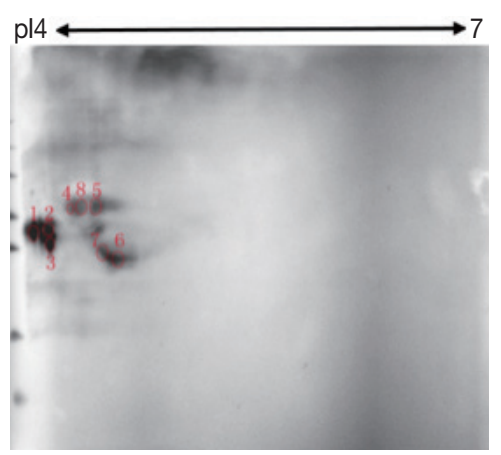

C

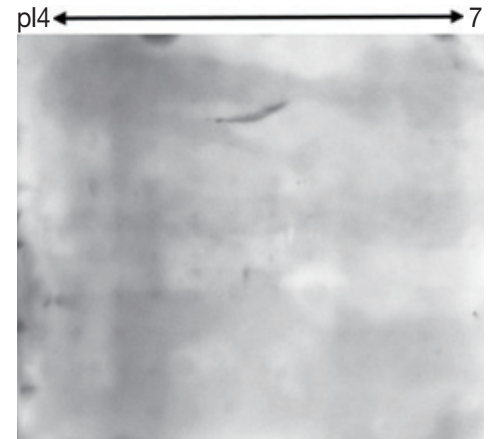

Fig. 1. Two-dimensional (2-D) gel and western blots of soluble extract from S. erinaceieuropaei plerocercoids. (A) 2-D gel stained with Coomassie blue R-250. Molecular mass markers (in kDa) are given on the left, and pl values are indicated. (B) A representative western blot incubated with pooled sera of sparganosis patients. Red circles are positive spots. (C) Western blot incubated with sera free from $S$. erinaceieuropaei infection.

Table 1. Annotation to the proteins of the immunoreactive spots against NCBI non-redundant protein database (Spirometra erinaceieuropaei)

\begin{tabular}{|c|c|c|c|c|c|c|c|}
\hline Spot & Protein name & MS & MP & Seq & SC (\%) & emPAl & $\mathrm{Mr}(\mathrm{kDa}) / \mathrm{pl}$ \\
\hline \multirow[t]{2}{*}{1} & Annexin E1 & 609 & 29 & 14 & 40.7 & 4.22 & $40.43 / 6.28$ \\
\hline & 14-3-3 protein & 249 & 9 & 5 & 18.9 & 1.14 & $28.94 / 5.10$ \\
\hline \multirow[t]{2}{*}{2} & Annexin E1 & 1,221 & 43 & 19 & 61.6 & 12.41 & $40.43 / 6.28$ \\
\hline & Succinate-CoA ligase & 221 & 11 & 6 & 15.4 & 1.3 & $34.34 / 9.19$ \\
\hline \multirow[t]{2}{*}{3} & 14-3-3 protein & 437 & 14 & 8 & 34.3 & 2.31 & $28.94 / 5.10$ \\
\hline & 14-3-3-like protein 2 & 280 & 7 & 4 & 16.1 & 0.75 & $28.17 / 5.23$ \\
\hline \multirow[t]{2}{*}{4} & Glycine C-acetyltransferase & 219 & 7 & 6 & 16.4 & 0.62 & $46.08 / 8.89$ \\
\hline & Deoxyribonuclease & 202 & 6 & 6 & 21.1 & 0.7 & $35.83 / 6.93$ \\
\hline \multirow[t]{2}{*}{6} & Plerocercoid growth factor/cysteine protease & 807 & 24 & 3 & 11.6 & 7.5 & $38.27 / 7.5$ \\
\hline & Cysteine proteinase, partial & 760 & 23 & 2 & 9.5 & 4.66 & $20.76 / 4.66$ \\
\hline \multirow[t]{3}{*}{8} & 14-3-3-like protein 2 & 269 & 8 & 6 & 26.1 & 5.23 & $28.17 / 5.23$ \\
\hline & Glycine C-acetyltransferase & 226 & 7 & 5 & 12.6 & 0.62 & $46.08 / 8.89$ \\
\hline & Deoxyribonuclease & 204 & 6 & 6 & 19.9 & 0.7 & $35.83 / 6.93$ \\
\hline
\end{tabular}

MS, mascot score; MP, matched peptide; Seq, sequence; SC, sequence coverage (\%); emPAl, exponentially modified protein abundance index; Mr $(\mathrm{kDa}) / \mathrm{pl}$, theoretical molecular weight/isoelectric point.

6 spots in the proteome of S. erinaceieuropaei plerocercoids based on LC-MS/MS results. The annexin E1 was identified in 2 spots (Spot No.: 1, 2). The 14-3-3 protein, 14-3-3-like protein 2, glycine C-acetyltransferase and deoxyribonuclease were also found in 2 spots (Table 1). Other proteins such as succinateCoA ligase, plerocercoid growth factor/cysteine protease and cysteine proteinase (partial) were found in single spots. Compared the gel and LC-MS/MS results, spot 1 and spot 2, spot 4 and spot 5 and spot 8 , were 2 different groups of isoforms having similar molecular weight and different isoelectric points. The identified proteins were registered in NCBI protein database under accession numbers ADM26238.1, AGC74030.1, AFX 72983.1, AGC74035.1, AFX72991.1, AFX73002.1, BAB62718.1, BAB62816.1.

\section{Ontological identification}

The 8 identified proteins participated in biological processes, 3 in cellular components and 8 in molecular functions (Fig. 2). Molecular functions were involvement in hydrolase activity (annexin E1, cysteine protease and cysteine proteinase, partial), peptidase activity (cysteine protease and cysteine proteinase, partial), transferase activity (glycine C-acetyltransferase, 14-3-3 protein and 14-3-3-like protein 2), ligase activity (succinate-CoA ligase) and helicase activity (annexin E1). Another protein was a calcium ion binding protein involved in ribosome biogenesis and biosynthetic process (deoxyribonuclease). Seven proteins were assigned to metabolic process: annexin E1, succinate-CoA ligase, glycine C-acetyltransferase, 143-3 protein, 14-3-3-like protein 2, cysteine protease and cyste- 


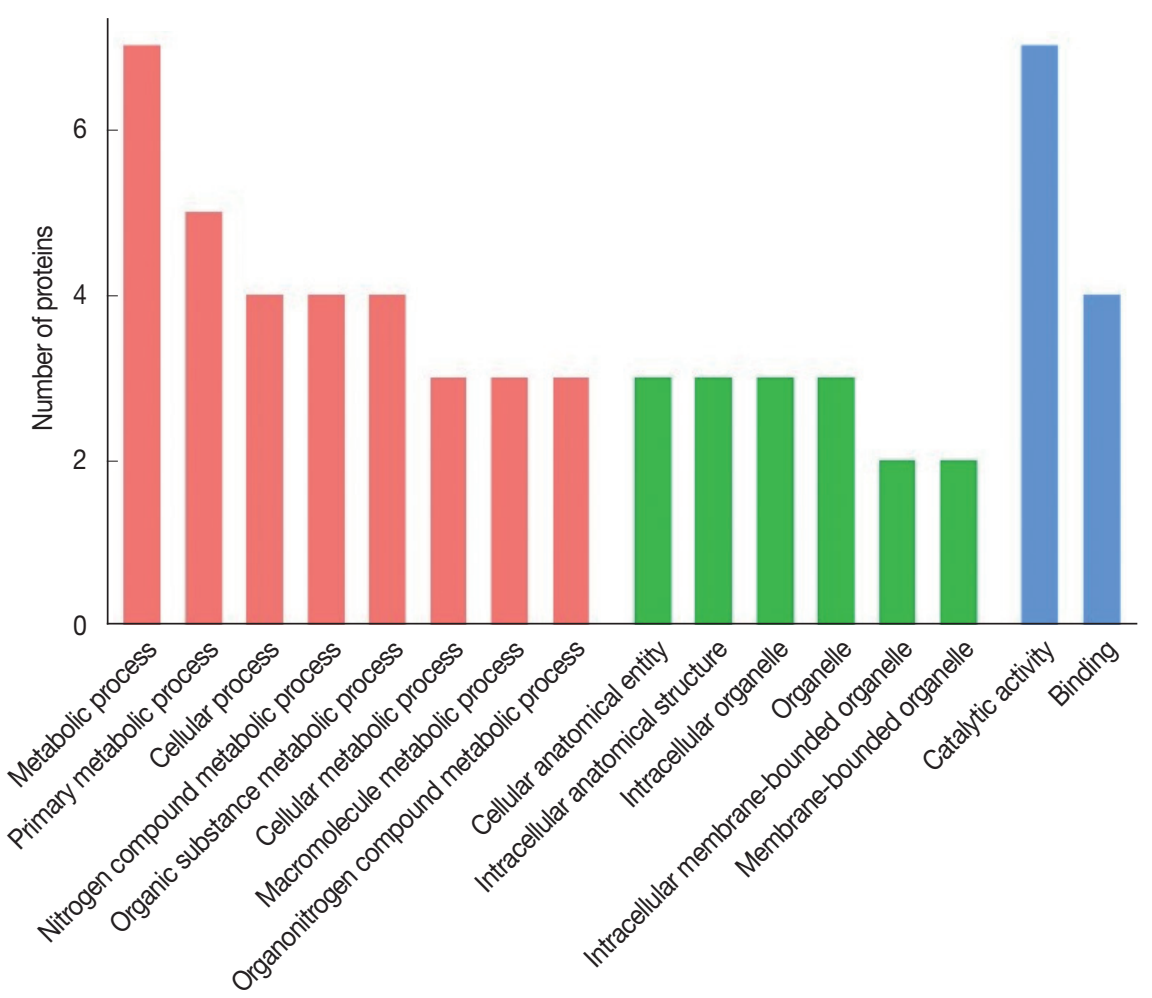

Fig. 2. Gene Ontology of the S. erinaceieuropaei plerocercoid immunogenic proteins.

A

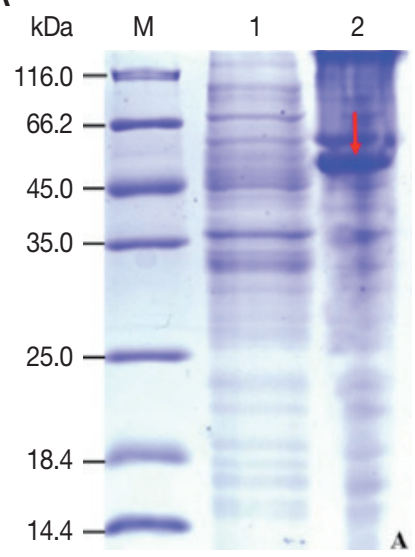

B

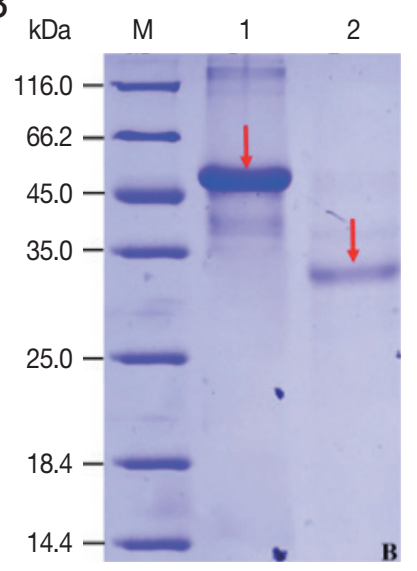

C

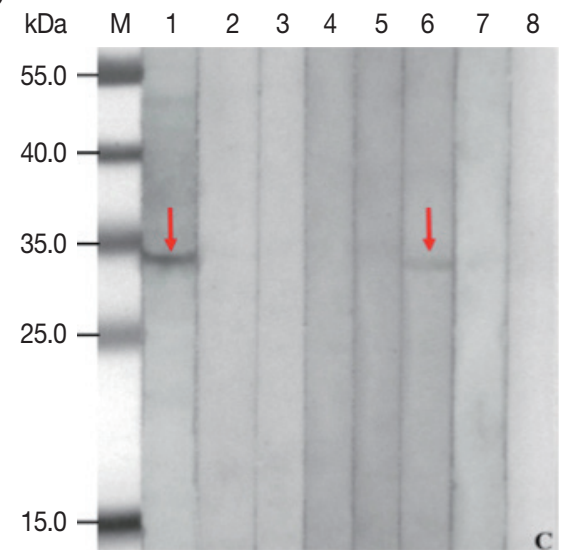

Fig. 3. Expression, purification and seroreactivity of rCP. (A) Expression of rCP. Lane 1, uninduction; Lane 2, induced. (B) Purification of rCP. Lane 1, purified fusion protein; Lane 2, purified rCP. (C) Immunoblot of rCP to sera from parasitic infections. Lane 1, sparganosis; Lane 2, echinococcosis; Lane 3, cysticercosis; Lane 4, trichinellosis; Lane 5, schistosomiasis; Lane 6, clonorchiasis; Lane 7, paragonimiasis; Lane 8, normal control. Target bands are marked with red arrows. Lane M, molecular marker (kDa).

ine proteinase (partial), while 4 proteins (annexin E1, succinate-CoA ligase, glycine C-acetyltransferase and deoxyribonuclease) participated in the cellular process.

\section{Immunoreactivity of recombinant protein}

The rCP was expressed in E. coli as a fusion protein with molecular weight of about $51 \mathrm{KDa}$ (Fig. 3A). After purified and cleaved off SUMO-tag, the purified rCP was about $35 \mathrm{kDa}$ consistent with predicted molecular weight (Fig. 3B). The rCP was recognized by the sera of sparganosis patients, while weakly recognized by sera of clonorchiasis patients. Other sera of patients with echinococcosis, cysticercosis, trichinellosis, schistosomiasis and paragonimiasis did not show any detectable reaction. Sera of normal control also did not show any 
positive reaction (Fig. 3C). The results indicated that $\mathrm{rCP}$ shows a specific immunoreactivity and the potential value for serodiagnosis of sparganosis.

\section{DISCUSSION}

In this study, 8 antigenic proteins were identified. Among these proteins that may serve as potential diagnostic antigens, the highest sequence coverage was obtained for annexin E1. Annexins are a large family of calcium-dependent phospholipid-binding proteins which are widely distributed across all eukaryotes. It plays important roles in multiple functions, such as calcium metabolism, cell anti-inflammatory activity, membrane repair and membrane transport, and probably participate in cell adhesion, proliferation, differentiation, and apoptosis $[38,39]$ because of their ability to bind phospholipids and proteins. According to differences in gene location and protein structure, annexins are divided into 6 categories, which includes 'A' (from vertebrates), 'B' (invertebrates), 'C' (fungi), 'D' (plants), 'E' (protists) and 'F'(bacteria) annexins [39-41]. Parasite annexins have been found to play critical roles in mechanisms linked to their survival, including the maintenance of cell structure integrity by attaching the cytoskeleton, modulation of the immune responses of the vertebrate hosts and vesicular transport $[41,42]$. For cestoda, annexin B1 was found in the cyst stage of Taenia solium whose metacestode can cause cysticercosis. By binding to the eosinpohils at the site of inflammation and causing the apoptosis, the immune response is reduced and keep T. solium survive $[43,44]$. Furthermore, the recombinant annexin B30, annexin B5a, annexin B7a and annexin B5b from Schistosoma mansoni were immunolocalized to the tegument, with immunoreactivity also occurring in cells and in muscle of adult parasite, and could be useful candidates for vaccine or diagnostic development [45]. Annexin E1 was previously identified from a cDNA library of $S$. erinaceieuropaei adult worm [46]. The structure and function analysis of this protein showed that it might be a desirable molecular target for immunological tests. Our study also indicated that annexin E1 of $S$. erinaceieuropaei recognized by patient sera might be the diagnostic antigen for sparganosis, which needed to be further confirmed.

Another potential S. erinaceieuropaei antigen identified in the present study, 14-3-3 protein, was previously identified in several unicellular and multicellular parasites [47]. The 14-3-3 proteins are a family of highly conserved proteins that are widely expressed in many eukaryotic organisms and has been shown to play a role in metabolism, signal transduction networks, stress conditions, cell cycle regulation, control of apoptosis and cellular organelle trafficking [48-50]. In the field of parasitology, 14-3-3 protein has been assumed to play an important role in parasite proliferation and survival $[47,51]$. Various structures and functions of 14-3-3 protein have been studied in Toxoplasma gondii [52], S. japonicum [53], Echinococcus granulosus [54], Fasciola gigantic [55], Clonorchis sinensis [56], Opisthorchis viverrini [57], Trichinella britovi [58] and Strongyloides stercoralis [59]. In S. japonicum, a sandwich time-resolved fluoroimmunoassay (TRFIA) for detecting the circulating antigen 14-3-3 has been developed, and has demonstrated to be a good potential diagnostic method for schistosomiasis [53]. The recombinant $F$. gigantica 14-3-3e protein has been proved to be involved in $F$. gigantica recognition by innate immune cells, and may have applications for development of diagnostics and therapeutic interventions [55]. For E. granulosus, recombinant 14-3-3 protein (rEg14-3-3) was verified to possess antigenicity and immunogenicity, and mice vaccinated with rEg14-3-3 and challenged with protoscoleces revealed significant protective immunity of $84.47 \%$. The result indicate that rEg14-3-3 have the potential to be applied as a vaccine against E. granulosus [60]. However, the characterization and immunomodulatory effects of S. erinaceieuropaei 14-3-3 proteins have not yet been reported.

Additionally, cysteine proteinase was also detected in the present study, which was previously identified from the crude and excretory-secretory antigens of $S$. erinaceieuropaei plerocercoids [35,36]. Cysteine proteinase (CP) is a type of protein hydrolase that has cysteine residues in the active center of the enzyme and plays a principal role in the development and survival of parasites, including modulation of the immune system of the host, participation in parasite virulence and invasion [61]. CP with different molecular weights ( $53 \mathrm{kDa}, 36 \mathrm{kDa}, 27$ $\mathrm{kDa}$, and $21 \mathrm{kDa}$ ) has been found in S. erinaceieuropaei plerocercoid soluble antigens, and the $36 \mathrm{kDa}$ protein is the main antigenic component of the ES proteins [62-65]. Later study showed that the $36 \mathrm{kDa}$ S. erinaceieuropaei cysteine protease (SeCP) was a plerocercoid stage-specific protein located in the teguments and parenchymal tissue. The recombinant SeCP ( $\mathrm{SeCP}$ ) had cysteine protease activity and functioned to degrade host proteins. The sensitivity of rSeCP-ELISA was 100\% when performed on sera of patients with sparganosis, while the specificity of rSeCP was $98.22 \%$ (166/169) [66]. In this 
study, rCP encoding a $35 \mathrm{kDa}$ protein from plerocercoids was successfully expressed and purified using the pET SUMO vector system. On western blotting analysis, rCP reacted strongly with the sera of sparganosis patients and only invoked serological cross reactions with clonorchiasis. The result demonstrated that rCP might be a potential diagnostic biomarker for S. erinaceieuropaei plerocercoids infection, and further studies were needed to confirm that. These researches indicated that our strategy using immunoproteomic analysis is useful for discovering antigenic proteins of S. erinaceieuropaei plerocercoids.

So far few of candidates for serodiagnosis have been cloned, include the cysteine proteinase [66], antigenic polypeptide [67] and translationally controlled tumor protein [68]. Hence, there is still a need to intensively study of $S$. erinaceieuropaei to identify which molecules initiate the immune response in the host. Our research on S. erinaceieuropaei antigens aimed at identifying elements related to host-parasite interactions, thus serving as a substantial basis in the search for potential diagnostic markers or drug targets.

\section{ACKNOWLEDGMENTS}

This work was supported by the General Program Shanghai Municipal Commission of Health and Family Planning of China (grant no. 201740076), the National Parasite Resource Bank (grant no. NPRC-2019-194-30), the Open Project of Key Laboratory of Parasite and Vector Biology, China Ministry of Health (grant no. WSBKFKT-201804, WSBKFKT-201806).

\section{CONFLICT OF INTEREST}

The authors declare that they have no competing interests.

\section{REFERENCES}

1. Liu Q, Li MW, Wang ZD, Zhao GH, Zhu XQ. Human sparganosis, a neglected food borne zoonosis. Lancet Infect Dis 2015; 15 : 1226-1235. https://doi.org/10.1016/S1473-3099(15)00133-4

2. Li MW, Song HQ, Li C, Lin HY, Xie WT, Lin RQ, Zhu XQ. Sparganosis in mainland China. Int J Infect Dis 2011; 15: 154-156. https://doi.org/10.1016/j.ijid.2010.10.001

3. Kim JG, Ahn CS, Sohn WM, Nawa Y, Kong Y. Human sparganosis in Korea. J Korean Med Sci 2018; 33: e273. https://doi.org/10.3346/ jkms.2018.33.e273

4. Magnino S, Colin P, Dei-Cas E, Madsen M, McLauchlin J, Nöckler K, Maradona MP, Tsigarida E, Vanopdenbosch E, Peteghem CV. Biological risks associated with consumption of reptile products.
Int J Food Microbiol 2009; 134: 163-175. http://doi.org/10.1016/ j.ijfoodmicro.2009.07.001

5. Jeon HK, Park H, Lee D, Choe S, Kim KH, Huh S, Sohn WM, Chai JY, Eom KS. Human infections with Spirometra decipiens plerocercoids identified by morphologic and genetic analyses in Korea. Korean J Parasitol 2015; 53: 299-305. http://doi.org/10.3347/ kjp.2015.53.3.299

6. Anantaphruti MT, Nawa Y, Vanvanitchai Y. Human sparganosis in Thailand: an overview. Acta Trop 2011; 118: 171-176. http:// doi.org/10.1016/j.actatropica.2011.03.011

7. Wiwanitkit V. A review of human sparganosis in Thailand. Int J Infect Dis 2005; 9: 312-316. http://doi.org/10.1016/j.ijid.2004. 08.003

8. Yamasaki H, Sanpool O, Rodpai R, Sadaow L, Laummaunwai $\mathrm{P}$, Un $\mathrm{M}$, Thanchomnang T, Laymanivong $\mathrm{S}$, Aung WPP, Intapan PM, Maleewong W. Spirometra species from Asia: genetic diversity and taxonomic challenges. Parasitol Int 2021; 80: 102181. http:// doi.org/10.1016/j.parint.2020.102181

9. Kudo T, Fujioka A, Korenaga M, Yamasaki H, Morishima Y, Sugiyama H, Nakajima H, Sano S. Molecular identification of intramuscular and subcutaneous Spirometra erinaceiuropaei sparganosis in a Japanese patient. J Dermatol 2017; 44: 138-139. https:// doi.org/10.1111/1346-8138.13739

10. Cobbold TS. Description of Ligula mansoni, a new human cestode. Zool J Linn Soc Lond 1883; 17: 78-83. https://doi.org/10.1111/ j.1096-3642.1883.tb00237.x

11. Zhang X, Hong X, Liu SN, Jiang P, Zhao SC, Sun CX, Wang ZQ, Cui J. Large-scale survey of a neglected agent of sparganosis Spirometra erinaceieuropaei (Cestoda: Diphyllobothriidae) in wild frogs in China. PLoS Negl Trop Dis 2020; 14: e0008019. http:// doi.org/10.1371/journal.pntd.0008019

12. Cui J, Lin XM, Zhang HW, Xu BL, Wang ZQ. Sparganosis, Henan Province, central China. Emerg Infect Dis 2011; 17: 146-147. http:// doi.org/10.3201/eid1701.101095

13. Xia Q, Yan J. Diagnosis and surgical management of orbital sparganosis. Eye (Lond) 2019; 33: 1418-1422. http://doi.org/10.1038/ s41433-019-0427-x

14. Kazemi A, Awosika O, Burgess C. Subcutaneous Sparganosis of the Breast. J Clin Aesthet Dermatol 2018; 11: 26-27.

15. Hwang JM, Hwang DS, Kang C, Lee JW. Subcutaneous sparganosis mimicking soft tissue tumor: a case report. Int Med Case Rep J 2019; 12: 47-50. http://doi.org/10.2147/IMCRJ.S192764

16. Otranto D, Eberhard ML. Zoonotic helminths affecting the human eye. Parasit Vectors 2011; 4: 41. http://doi.org/10.1186/17563305-4-41

17. Hong D, Xie H, Zhu M, Wan H, Xu R, Wu Y. Cerebral sparganosis in mainland Chinese patients. J Clin Neurosci 2013; 20: 1514-1519. http://doi.org/10.1016/j.jocn.2012.12.018

18. Zhang P, Zou Y, Yu FX, Wang Z, Lv H, Liu XH, Ding HY, Zhang TT, Zhao PF, Yin HX, Yang ZH, Wang ZC. Follow-up study of high-dose praziquantel therapy for cerebral sparganosis. PLoS Negl Trop Dis 2019; 13: e0007018. http://doi.org/10.1371/journal.pntd.0007018 
19. Cui J, Wang Y, Zhang X, Lin XM, Zhang HW, Wang ZQ, Chen JX. A neglected risk for sparganosis: eating live tadpoles in central China. Infect Dis Poverty 2017; 6: 58. http://doi.org/10.1186/ s40249-017-0265-7

20. Murata K, Abe T, Gohda M, Inoue R, Ishii K, Wakabayashi Y, Kamida T, Fujiki M, Kobayashi H, Takaoka H. Difficulty in diagnosing a case with apparent sequel cerebral sparganosis. Surg Neurol 2007; 67: 409-411. http://doi.org/10.1016/j.surneu.2006.06.056

21. Song T, Wang WS, Zhou BR, Mai WW, Li ZZ, Guo HC, Zhou F. CT and MR characteristics of cerebral sparganosis. Am J Neuroradiol 2007; 28: 1700-1705. http://doi.org/10.3174/ajnr.A0659

22. Liao HY, Li DT, Zhou B, Liu J, Li YJ, Liu H, Wu YZ, Zhu XZ, Tan CL. Imaging characteristics of cerebral sparganosis with live worms. J Neuroradiol 2016; 43: 378-383. http://doi.org/10.1016/ j.neurad.2016.08.001

23. Lee JH, Yu JS, Park MS, Lee SI, Yang SW. Abdominal sparganosis presenting as an abscess with fistulous communication to the bowel. Am J Roentgenol 2005; 185: 1084-1085. http://doi.org/10.2214/ AJR.04.1680

24. Koo JH, Cho WH, Kim HT, Lee SM, Chung BS, Joo CY. A case of sparganosis mimicking a varicose vein. Korean J Parasitol 2006; 44: 91-94. http://doi.org/10.3347/kjp.2006.44.1.91

25. Rengarajan S, Nanjegowda N, Bhat D, Mahadevan A, Sampath S, Krishna S. Cerebral sparganosis: a diagnostic challenge. Br J Neurosurg 2008; 22: 784-786. http://doi.org/10.1080/02688690802088073

26. Kong Y, Kang SY, Cho SY. Single step purification of potent antigenic protein from sparganum by gelatin-affinity chromatography. Kisaengchunghak Chapchi 1991; 29: 1-7. http://doi.org/10.3347/ kjp.1991.29.1.1

27. Nishiyama T, Ide T, Himes SR Jr., Ishizaka S, Araki T. Immunodiagnosis of human sparganosis mansoni by micro-chemiluminescence enzyme-linked immunosorbent assay. Trans R Soc Trop Med Hyg 1994; 88: 663-665. http://doi.org/10.1016/0035-9203 (94)90218-6

28. Cui J, Li N, Wang ZQ, Jiang P, Lin XM. Serodiagnosis of experimental sparganum infections of mice and human sparganosis by ELISA using ES antigens of Spirometra mansoni spargana. Parasitol Res 2011; 108: 1551-1556. http://doi.org/10.1007/s00436010-2206-2

29. Yeo IS, Yong TS, Im K. Serodiagnosis of human sparganosis by a monoclonal antibody-based competition ELISA. Yonsei Med J 1994; 35: 43-48. http://doi.org/10.3349/ymj.1994.35.1.43

30. Ju C, Xu B, Lu Y, Mo XJ, Zhang T, Chen SB, Liu F, Cui SJ, Liu W, Chen JH, Feng Z, Peng JX, Hu W. Comparative immunomic analysis of Schistosoma japonicum soluble egg antigens reacting with patient sera before and after praziquantel treatment. J Anim Vet Adv 2012; 11: 2828-2838. http://doi.org/10.3923/javaa.2012.2828.2838

31. Acevedo N, Mohr J, Zakzuk J, Samonig M, Briza P, Erler A, Pomés A, Huber CG, Ferreira F, Caraballo L. Proteomic and immunochemical characterization of glutathione transferase as a new allergen of the nematode Ascaris lumbricoides. PLoS One 2013; 8: e78353. http://doi.org/10.1371/journal.pone.0078353

32. Grzelak S, Stachyra A, Bien-Kalinowska J. The first analysis of
Trichinella spiralis and Trichinella britovi adult worm excretory-secretory proteins by two-dimensional electrophoresis coupled with LC-MS/MS. Vet Parasitol 2021: 109096. http://doi.org/10.1016/ j.vetpar.2020.109096

33. Sun XM, Ji YS, Elashram SA, Lu ZM, Liu XY, Suo X, Chen QJ, Wang H. Identification of antigenic proteins of Toxoplasma gondii $\mathrm{RH}$ strain recognized by human immunoglobulin G using immunoproteomics. J Proteomics 2012; 77: 423-432. http://doi.org/10.1016/ j.jprot.2012.09.018

34. Xu B, Liu XF, Cai YC, Huang JL, Zhang RX, Chen JH, Chen XJ, Zhou X, Xu XN, Zhou Y, Zhang T, Chen SB, Li J, Wu QF, Sun CS, Fu YF, Chen JX, Zhou XN, Hu W. Screening for biomarkers reflecting the progression of Babesia microti infection. Parasit Vectors, 2018; 11: 379. http://doi.org/10.1186/s13071-018-2951-0

35. Hu DD, Cui J, Xiao D, Wang L, Liu LN, Liu RD, Zhang JZ, Wang ZQ. Identification of early diagnostic antigens from Spirometra erinaceieuropaei sparganum soluble proteins using immunoproteomics. Southeast Asian J Trop Med Public Health 2014; 45: 576-583.

36. Hu DD, Cui J, Wang L, Liu LN, Wei T, Wang ZQ. Immunoproteomic Analysis of the Excretory-Secretory Proteins from Spirometra mansoni Sparganum. Iran J Parasitol 2013; 8: 408-416.

37. Lu Y, Chen JX, Li H, Cai YC, Ai L, Chu YH, Song P, Chen SH. Infection of Spirometra mansoni plerocercoid in snakes from Shanghai Zoo. Chin J Parasitol Parasit Dis 2018; 36: 593-596 (in Chinese).

38. Schloer S, Pajonczyk D, Rescher U. Annexins in translational research: hidden treasures to be found. Int J Mol Sci 2018; 19: 1781. http://doi.org/10.3390/ijms19061781

39. Cantacessi C, Seddon JM, Miller TL, Leow CY, Thomas L, Mason L, Willis C, Walker G, Loukas A, Gasser RB, Jones MK, Hofmann A. A genome-wide analysis of annexins from parasitic organisms and their vectors. Sci Rep 2013; 3: 2893. http://doi.org/10.1038/ srep02893

40. Moss SE, Morgan RO. The annexins. Genome Biol 2004; 5, 219. https://doi.org/10.1186/gb-2004-5-4-219

41. Hofmann A, Osman A, Leow CY, Driguez P, McManus DP, Jones MK. Parasite annexins--new molecules with potential for drug and vaccine development. Bioessays, 2010; 32: 967-776. http:// doi.org/10.1002/bies.200900195

42. Gerke V, Moss SE. Annexins: from structure to function. Physiol Rev 2002; 82: 331-371. http://doi.org/10.1152/physrev.00030.2001

43. Zhang Y, Wang KH, Guo YJ, Lu YM, Yan HL, Song YL, Wang F, Ding FX, Sun SH. Annexin B1 from Taenia solium metacestodes is a newly characterized member of the annexin family. Biol Chem 2007; 388: 601-610. http://doi.org/10.1515/BC.2007.071

44. Yan HL, Xue G, Mei Q, Ding FX, Wang YZ, Sun SH. Calcium-dependent proapoptotic effect of Taenia solium metacestodes annexin B1 on human eosinophils: a novel strategy to prevent host immune response. Int J Biochem Cell Biol 2008; 40: 2151-2163. http://doi.org/10.1016/j.biocel.2008.02.018

45. Leow CY, Willis C, Leow CH, Hofmann A, Jones M. Molecular characterization of Schistosoma mansoni tegument annexins and 
comparative analysis of antibody responses following parasite infection. Mol Biochem Parasitol 2019; 234: 111231. https://doi. org/10.1016/j.molbiopara.2019.111231

46. Wu Q, Li DD, Ma PP, Lv G. Application of bioinformatic analysis in Annexin E1 of Spirometra erinaceieuropaei. J Hainan Med Coll 2010; 16: 1106-1110 (in Chinese).

47. Siles-Lucas Mdel M, Gottstein B. The 14-3-3 protein: a key molecule in parasites as in other organisms. Trends Parasitol 2003; 19: 575-581. http://doi.org/10.1016/j.pt.2003.10.003

48. Liu J, Giri BR, Chen Y, Cheng G. 14-3-3 protein and ubiquitin C acting as SjIAP interaction partners facilitate tegumental integrity in Schistosoma japonicum. Int J Parasitol 2019; 49: 355-364. http:// doi.org/10.1016/j.ijpara.2018.11.011

49. Gardino AK, Yaffe MB. 14-3-3 proteins as signaling integration points for cell cycle control and apoptosis. Semin Cell Dev Biol 2011; 22: 688-695. http://doi.org/10.1016/j.semcdb.2011.09.008

50. Obsilova V, Kopecka M, Kosek D, Kacirova M, Kylarova S, Rezabkova L, Obsil T. Mechanisms of the 14-3-3 protein function: regulation of protein function through conformational modulation. Physiol Res 2014; 63 (suppl): 155-164. http://doi.org/10.33549/ physiolres.932659

51. Stevers LM, Sijbesma E, Botta M, MacKintosh C, Obsil T, Landrieu I, Cau Y, Wilson AJ, Karawajczyk A, Eickhoff J, Davis J, Hann M, O'Mahony G, Doveston RG, Brunsveld L, Ottmann C. Modulators of 14-3-3 Protein-Protein Interactions. J Med Chem 2018; 61: 3755-3778. http://doi.org/10.1021/acs.jmedchem.7b00574

52. Li FC, Liu Q, Elsheikha HM, Yang WB, Hou JL, Zhu XQ. Identification of two novel host proteins interacting with Toxoplasma gondii 14-3-3 protein by yeast two-hybrid system. Parasitol Res 2018; 117: 1291-1296. http://doi.org/10.1007/s00436-018-5812-z

53. Qian CY, Huang B, Yu CX, Zhang J, Yin XR, Wang J, Song LJ, Zhang $\mathrm{W}, \mathrm{Ke} \mathrm{XD}$. Detection of the circulating antigen 14-3-3 protein of Schistosoma japonicum by time-resolved fluoroimmunoassay in rabbits. Parasit Vectors 2011; 4: 95. http://doi.org/10.1186/1756-33054-95

54. Teichmann A, Vargas DM, Monteiro KM, Meneghetti BV, Dutra CS, Paredes R, Galanti N, Zaha A, Ferreira HB. Characterization of 14-3-3 isoforms expressed in the Echinococcus granulosus pathogenic larval stage. J Proteome Res 2015; 14: 1700-1715. http://doi. org/10.1021/pr5010136

55. Tian AL, Lu M, Calderon-Mantilla G, Petsalaki E, Dottorini T, Tian XW, Wang YJ, Huang SY, Hou JL, Li XR, Elisheikha HM, Zhu XQ. A recombinant Fasciola gigantica 14-3-3 epsilon protein (rFg14-3-3e) modulates various functions of goat peripheral blood mononuclear cells. Parasit Vectors 2018; 11: 152. http:// doi.org/10.1186/s13071-018-2745-4

56. Lee MR, Kim YJ, Kim DW, Yoo WG, Cho SH, Hwang KY, Ju JW, Lee WJ. The identification of antigenic proteins: 14-3-3 protein and propionyl-CoA carboxylase in Clonorchis sinensis. Mol Biochem Parasitol 2012; 182: 1-6. http://doi.org/10.1016/j.molbiopara.2011.11.006
57. Kafle A, Puchadapirom P, Plumworasawat S, Dontumprai R, Chan-On W, Buates S, Laha T, Sripa B, Suttiprapa S. Identification and characterization of protein 14-3-3 in carcinogenic liver fluke Opisthorchis viverrini. Parasitol Int 2017; 66: 426-431. http:// doi.org/10.1016/j.parint.2016.10.021

58. Stachyra A, Grzelak S, Basalaj K, Zawistowska-Deniziak A, BieńKalinowska J. immunization with a recombinant protein of Trichinella britovi 14-3-3 triggers an immune response but no protection in mice. Vaccines (Basel) 2020; 8: 515. http://doi.org/10.3390/vaccines 8030515

59. Masoori L, Falak R, Mokhtarian K, Bandehpour M, Razmjou E, Jalallou N, Jafarian F, Akhlaghi L, Meamar AR. Production of recombinant 14-3-3 protein and determination of its immunogenicity for application in serodiagnosis of strongyloidiasis. Trans $\mathrm{R}$ Soc Trop Med Hyg 2019; 113: 326-331. http://doi.org/10.1093/ trstmh/trz006

60. Li ZJ, Wang YN, Wang Q, Zhao W. Echinococcus granulosus 14-3-3 protein: a potential vaccine candidate against challenge with Echinococcus granulosus in mice. Biomed Environ Sci 2012; 25: 352-358. http://doi.org/10.3967/0895-3988.2012.03.014

61. Siqueira-Neto JL, Debnath A, McCall LI, Bernatchez JA, Ndao M, Reed SL, Rosenthal PJ. Cysteine proteases in protozoan parasites. PLoS Negl Trop Dis 2018; 12: e0006512. http://doi.org/10.1371/ journal.pntd.0006512

62. Song CY, Chappell CL. Purification and partial characterization of cysteine proteinase from Spirometra mansoni plerocercoids. J Parasitol 1993; 79: 517-524. http://doi.org/10.2307/3283376

63. Fukase T, Matsuda Y, Akihama S, Itagaki H. Purification and some properties of cysteine protease of Spirometra erinacei plerocercoid (Cestoda: Diphyllobothriidae). Jpn J Parasitol 1985; 34, 351-360.

64. Kong Y, Kang SY, Kim SH, Chung YB, Cho SY. A neutral cysteine protease of Spirometra mansoni plerocercoid invoking an IgE response. Parasitology 1997; 114: 263-271. http://doi.org/10.1017/ s0031182096008529

65. Cho SY, Chung YB, Kong Y. Component proteins and protease activities in excretory-secretory product of sparganum. Korean J Parasitol 1992; 30: 227-230. http://doi.org/10.3347/kjp.1992.30.3.227

66. Liu LN, Wang ZQ, Zhang X, Jiang P, Qi X, Liu RD, Zhang ZF, Cui J. Characterization of Spirometra erinaceieuropaei Plerocercoid Cysteine Protease and Potential Application for Serodiagnosis of Sparganosis. PLoS Negl Trop Dis 2015; 9: e0003807. http://doi. org/10.1371/journal.pntd.0003807

67. Cui J, Wei T, Liu LN, Zhang X, Qi X, Zhang ZF, Wang ZQ. Molecular characterization of a Spirometra mansoni antigenic polypeptide gene encoding a 28.7 kDa protein. Parasitol Res 2014; 113: 3511-3516. http://doi.org/10.1007/s00436-014-4065-8

68. Lu YJ, Lu G, Shi DZ, Li LH, Zhong SF. Bioinformatic analysis for structure and function of TCTP from Spirometra mansoni. Asian Pac J Trop Med 2013; 6: 709-712. http://doi.org/10.1016/s19957645(13)60123-6 
